\title{
Computerized Drug Verification System: A Panacea for Effective Drug Verification
}

\author{
Oketa Christian Kelechi ${ }^{1}$, Alo Uzoma Rita ${ }^{2}$, Okemiri Henry Anayo ${ }^{3}$, Richard-Nnabu Nneka Ernestecia ${ }^{4}$ \\ Achi Ifeanyi Isaiah ${ }^{5}$, Chinazo I. Chima ${ }^{6}$, Afolabi Idris Yinka ${ }^{7}$, Mgbanya Praise Chinenye $^{8}$ \\ Department of Mathematics / Computer Science / Statistics / Informatics \\ Alex Ekwueme Federal University Ndufu Alike \\ Ebonyi State, Nigeria
}

\begin{abstract}
Computerized Drug Verification System (CDVS) is a research work geared towards establishing the means of identifying authentic drugs in Nigeria with emphasis on identifying the manufacturing date and expiration date respectively using an interactive mobile app. At the point of drug purchase, only few drugs have methods of verifying their authenticity using both Personal Identification Number (PIN) and National Agency for Food and Drug Administration Control (NAFDAC) number. However, the production and expiry dates of such drugs are not always known, hence drugs that have long expired may still report authentic thereby endangering the lives of the consumers. This research addresses the challenges providing an interactive platform for drug verification, with especially strength in its ability to incorporate the manufacturing date, the manufacturer, expiry date and the authentication of drugs. The product of this research, "NAFDAC VERIFY" an interactive mobile and web application can be used in verifying the authenticity of drugs in Nigeria in partnership with Mobile Authentication Service (MAS). This system which runs both on android mobile phones and web-based have been tested using some raw data and the system proves to be very robust and achieves the set out objectives.
\end{abstract}

Keywords-NAFDAC verify; security; drug verification; information system; drug authentication

\section{INTRODUCTION}

In, today's world, computer has permeated every part of life with its benefits. With this information processing which is the life wire of any organization is not left out in the scheme of computerization of Drug verification. Drugs are chemical substances that are administered to patients for curative purposes; it can also be called medicine because it is the essential part of people care. In recent years, rapid growth in fake drugs incident has claimed a lot of lives in Nigeria which led to the establishment of National Agency for Food and Drug Administration and Control (NAFDAC).

NAFDAC is a Nigerian government agency and they are responsible for controlling and regulating the manufacture, importation, exportation, advertisement, distribution, sale and use of food, drugs, cosmetics, medical devices, chemicals and prepackaged water. Its creation was inspired by a 1988 World Health Assembly resolution requesting countries to "help in combating the global health threat posed by counterfeit pharmaceuticals, and amidst growing concerns about the growing problem of fake and poorly regulated drugs in Nigeria" [1]. In 1989, over 150 children died due to error in a drug formulation. Consequent upon these problems, the Federal Government of Nigeria established NAFDAC to help create a fake-drug free environment in the country. NAFDAC's effort in this direction and in pursuance of the nation's vision for its establishment has been commendable, but researchers still wonder why there remain many unanswered questions: "why does Nigeria still have in existence open drug markets? Why do Nigerians in Drug business breech the stipulated drug laws and get away with it, and continue with their business, committing mass murder and smiling to their banks? How long are we to fight the battle of fake drug even with the threats on the lives of those who fight to preserve the health of the nation?" [1].

NAFDAC has a Mobile Authentication Service (MAS) that enables people to check whether a drug is original or fake with their mobile phones. NAFDAC Mobile Authentication Service (MAS) scratch and text authentication codes were launched in 2010 by NAFDAC's Director General, Dr. Paul Orhii to save consumers from the menace of fake drugs. However, the verification only shows that the drug in question is either authentic or fake without displaying the manufacturing and expiration dates respectively. NAFDAC MAS is not also enabled for all drugs as it is limited to certain drugs; mainly anti-malaria and antibiotic drugs. This research not provide a holistic solution to all the problems of drug faking in Nigeria. However, it carves a niche in providing means by which fake drugs and even genuine drugs which have expired can be tracked, knowing fully well that expired drugs are as harmful as their fake counterparts.

In Nigeria setting, a dubious drug manufacturer can preferably use the NAFDAC number of one drug for another, and the mode verification of drug authenticity does not include the status of the drug in respect to its manufacturing date and expiration date. Individuals who use these drugs are very reluctant to use verify the status of their drugs due to the non-interactive nature of the current drug verification system. At the point of drug purchase, there are no known methods of verifying; how genuine an assumed NAFDAC number is, the manufacturing date and expiry date of a drug. No working method to access NAFDAC database to know if a given NAFDAC number corresponds with the name of the drug in question. This research work is undertaken to uncover some of the problems with conventional drug reporting and verification systems. 


\section{StATEMENT OF THE PROBLEM}

In Nigeria setting, a dubious drug manufacturer can preferably use the NAFDAC number of one drug for another. At the point of drug purchase, there are no known methods of verifying; how genuine an assumed NAFDAC number is, including the manufacturing date and expiry date of drugs. No working method to access NAFDAC database to know if a given NAFDAC number corresponds with the name of the drug in question. This research work is undertaken to uncover some of the problems with conventional drug reporting and verification systems with emphasis on production dates and expiration dates of such drugs and beverages.

\section{OBJECTIVES OF THE STUDY}

In view of the problems mentioned above, the aim of this project is to design and implement a computerized drug verification system. With the following objectives in mind:

1) To develop an android application and a Web-based application to query the NAFDAC drug database for verification using verification pin.

2) To develop a web-based NAFDAC application for drug manufacturers registration, drug registration and verification pin generation.

3) To use a single pin (verification pin) to get complete information about a given drug.

4) To review literatures on the effects of drug faking on the Nigeria populace.

5) To create awareness on the negative implications of expired drugs and its unchecked circulations.

6) To carry out a SWOT analysis on the existing system in order to build a strong system that will stand the test of time.

\section{REVIEW OF RELATED LITERATURE}

Drug Faking is a worldwide health problem as the effects can be felt by both the manufacturing country of such drugs and the recipient countries. Olike, [1] noted that "National measures for combating of fake drugs in country might be insufficient because of the advanced sophistications of those who manufacture and sell them" [1]. Furthermore, Beverley Glass asserted that "product counterfeiting is increasing worldwide, both in terms of the volume, level of sophistication, and in the number the countries affected, both in the developed and developing world" [2]. Till date, Nigeria is not excluded in the challenges of fake drugs. Some people still prefer to undertake self-medication when they are ill, and often times the drugs are purchased from unlicensed drug vendors, whose drug quality is not certain. Moreover, fake drugs proved the main factor in contributing to high rate of deaths. "Over 150 children died in 1989 as a result of a formulation error in a drug. Such problems led to the establishment of NAFDAC, which would help create a fakedrug-free environment. The reason was to ensure effective registration of good quality drugs that are inexpensive in Nigeria. In April 2001, Professor Dora Akunyili the Director General of NAFDAC worked hard in combating the problems of sale of fake drugs" [1]. Dora Akunyili's comment on the menace of drugs faking and its negative impact on the Nigeria was captured properly by Olusegun, [3], "the problems of Fake drugs have embarrassed our healthcare providers and denied the confidence of the public on the nation's healthcare delivery system". The result of fake drug proliferation has led to treatment failures, organ dysfunction or damage, worsening of chronic disease conditions and the death of many Nigerians. The situation became so bad that even when patients were treated with genuine drugs, there were no response due to resistance caused by previous intake of fake drug " [4].

Fake drugs comes in variant forms and styles as observed by Buowari [4], "A counterfeit drug may contain inappropriate quantities of active ingredients, or none, may be improperly processed within the body (e.g., absorption by the body), may be supplied with inaccurate or fake packaging and labeling, or may contain ingredients that are not on the label (which may or may not be harmful). Medicines which are deliberately mislabeled to deceive consumers including genuine generic drugs but mislabeled are otherwise counterfeit. Counterfeit drugs are related to pharmacy fraud. Drug manufacturers and distributors are increasingly investing in countermeasures, such as traceability and authentication technologies, to try to minimize the impact of counterfeit drugs" [5].

The high occurrence of fake drugs in Nigeria is as a result of the arbitrary ways by which importation licenses on drugs were issued to people by then politicians and military leaders in the 80's, without considering the eventual implications their actions could bring on the health of the public. As the drug market boomed and competition for market share increased, some of the beneficiaries of drug importation licenses looked at the option of importing fake products in order to have a favorable margin over their competitors. "In Nigeria today, it is common knowledge that drugs are treated as general merchandise, which can be obtained easily from open markets, moving vehicles, faceless medicine stores, ferries, and even in the provision stores. This is because the drug distribution business has been left in the hands of non-professionals who just want to make profit at the expense of the consuming public. Poor people are faced with a confusing myriad of health providers and drug sellers" [1].

In February 2006, World Health Organization (WHO) established the first global partnership known as the International Medicinal Products Anti-Counterfeit Taskforce (IMPACT). This enabled them to create awareness and action in the fight against fake drugs. IMPACT focuses on key areas such as

- "Regulatory implementation approach to ensure that standards for quality, safety and efficacy are implemented and distribution chains effectively controlled.

- IMPACT will also help countries in monitoring borders through co-ordination of action between customs, police and the judiciary by working with the World Customs Agency, INTERPOL, and informal networks of enforcement officers. 
- Creating stronger legislation that will help empower those who deal with counterfeits and counterfeiters in the course of their work." [6].

However, improving International interaction especially to the developing world in order to identify the actual source of fake medicines tormenting their markets. IMPACT will also create International information networks to strengthen and monitor good trafficking, issue alerts from country to country.

\section{A. Technology Previously Used}

Most countries have developed strategic means of combating the prevalence of food and drug counterfeiting. The Mobile Authentication Service (MAS) works with mobile phones that are SMS enabled. It allows anyone with mobile phones to check whether drugs are fake or original without direct contact with the manufacturer. This service was introduced into Nigeria by National Agency for Food and Drug Administration and Control (NAFDAC) in response to the increasing rate of fake drugs distributed in the country. It is an attempt to turn mobile phones into tools that can help check this problem as many lives have been claimed and many are still endangered by the circulation of such fake products. The Mobile Authentication Service (MAS) uses Truscan technology which enables Consumers to input a twelve-digit number scripted on the back of the packaging of a drug and send the same via SMS to a dedicated number which returns a Negative or Positive response about the authenticity and genuineness of the product. This service was made possible through a partnership between NAFDAC, GSM operators and as well the representatives of national and international pharmaceutical companies in the Nigeria. Recently, the SMS drug verification was confirmed to be an efficient method in some Nigeria, but it is yet to be implemented on all drugs [7].

\section{THE M.A.S EXPERIENCE IN NIGERIA}

The National Agency for Food and Drug Administration and Control (NAFDAC) on February 2, 2010, launched the NAFDAC Mobile Authentication Service (MAS) with the aim of putting the power of checking for originality of product in the hands of consumer. The National Agency for food and Drug Administration and Control (NAFDAC) has made the implementation of MAS compulsory by all Pharmaceutical companies for all their major products especially the Antibiotics and the Anti-malarial drugs. The official deadline for implementation was set for $2^{\text {nd }}$ of March, 2013. It was hoped that this will be implemented and definitely allow Nigerians to check the authenticity of their medicines and eliminate fake drugs from circulation. To date it is clear that out of every 200 Nigerians only 1 knows about MAS representing $0.5 \%$ irrespective of the fact that the service is free. Well over a million text messages have been sent by Nigerians and millions of products now carry the unique sproxil label to show that such product is MAS ready (sproxil.com/mas.html). In February 2011, GlaxoSmithKline in partnership with National Agency for Food and Drug Administration and Control (NAFDAC), ran a six-month pilot anti-counterfeiting programme in Nigeria and in all, a total of 145,000 texts from 115,000 unique users were received. This figure represents approximately $10 \%$ of use from the total products sent out for the pilot programme. Ninety percent of text returned a genuine confirmation, $2.5 \%$ received a counterfeit alert and other received a message indicating a duplicate PIN.

\section{A. How to use NAFDAC MAS}

The step by step method that can be applied by the consumer even at the point of purchase of some selected drugs in order to verify their authenticity and genuineness via mobile phone are as follows:

1) "Scratch the sproxil label on the product to be purchased to reveal a unique 12-digit PIN.

2) Text the Unique 12-digit PIN as SMS to the short code 38353.

3) Immediately receive a response in form of SMS on your phone affirming the validity of the PIN either positively or negatively. With the support of all mobile network provider" [8] as seen in Fig. 1.

\section{B. Functions of NAFDAC in Nigeria}

1) Ensures compliance to standard: Ensuring compliance to standard is the major functions of the National Agency for Food and Drugs Administration Control (NAFDAC). Therefore what the National Agency for Food and Drugs Administration Control does is to carry out test in locally manufactured drugs and food and sometimes, those foods and likes imported into the country with the view to ensure that those products meet specified standard. There is designated standard specification to every product manufactured within Nigeria or imported into the country, there must be total consent by the council and until these product are approved by the council, such products is not consumable and it ensures effective quality control of these goods, such as food, and drugs, and cosmetics and medical devices, and packaged water, and chemicals, etc.

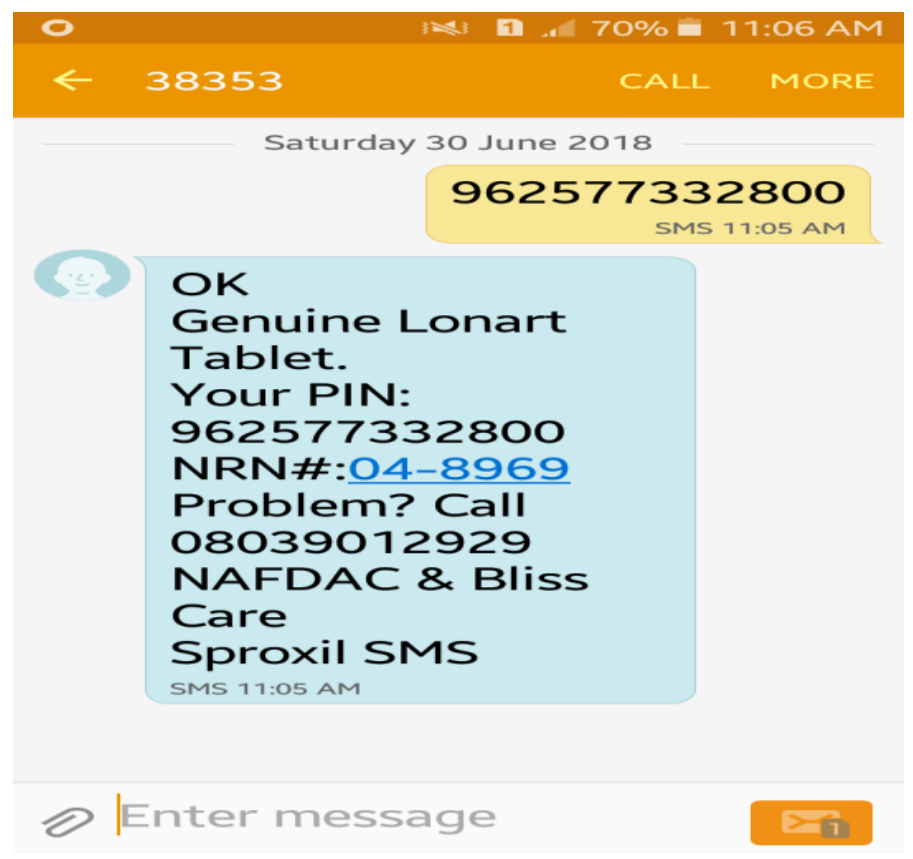

Fig. 1. Interface of NAFDAC MAS. 
2) Inspects imported food: Some of the goods imported into the country include, imported foods, imported drugs, imported cosmetics, imported bottle water, and imported chemical among others. NAFDAC ensures that what comes into the country meets the standard, the specification and make sure that there is an establishment of the relevant quality assurance system. They also ensure that, there is certification of the products and regulated product.

3) Investigates into production houses: Ensuring that premises, where these good are produced are in order and hygienic has been the primary function of the National Agency for Food and Drugs Administration Control. From time to time, the Agency will embarked on investigation tours to these places, where these goods are manufactured, and its investigation into these production houses is always appropriate and proper without leaving any stone unturned, the Agency will inspect and investigate raw material of these goods, be it drugs, food, cosmetics, etc. NAFDAC would check all accurately.

4) Registration of food and drug products: No products within this sector can thrive without National Agency for Food and Drugs Administration Control not knowing about such product, that's why NAFDAC itself has been registering these products.

5) Controls exportation: Exportation control is one of the function of the National Agency for Food and Drugs Administration Control, so far such product for exportation is product that is controlled and regulated by the National Agency for Food and Drugs Administration Control (NAFDAC). And they make sure that quality certification of all these products for exports are issued.

\section{ANALYSIS OF NAFDAC MAS}

The National Agency for Food and Drug Administration and Control (NAFDAC) established in January 1993, is responsible for regulating and controlling the manufacture, importation, exportation, advertisement, distribution, sale and use of food, drugs, cosmetics, medical devices, chemicals and packaged water. To achieve this aim, this agency has deployed several means such as demanding all manufactured drugs to be registered and assigned a NAFDAC registration number.

The next challenge facing drug end-users is how to verify the authenticity of a registration number imprinted on drugs. To achieve drug verification, the agency as well as other thirdparty organizations have to work in collaboration to achieve proper drug verification. On February 2, 2010, NAFDAC and Sproxil launched the NAFDAC Mobile Authentication Service (MAS), putting the power of product verification right in the hands of the consumer as shown in Fig. 2. MAS is powered by Sproxil's award-winning cloud-based Sproxil Defender ${ }^{\mathrm{TM}}$ technology, and remains the world's largest nation-wide implementation of consumer-facing SMS anticounterfeiting technology in the world (Sproxil®, 2018). Others including mPedigree, PharmaSecure etc, also provide drug verification alternatives to drug end-users in Nigeria (Nigerian Law Intellectual Property Watch Inc., 2018).

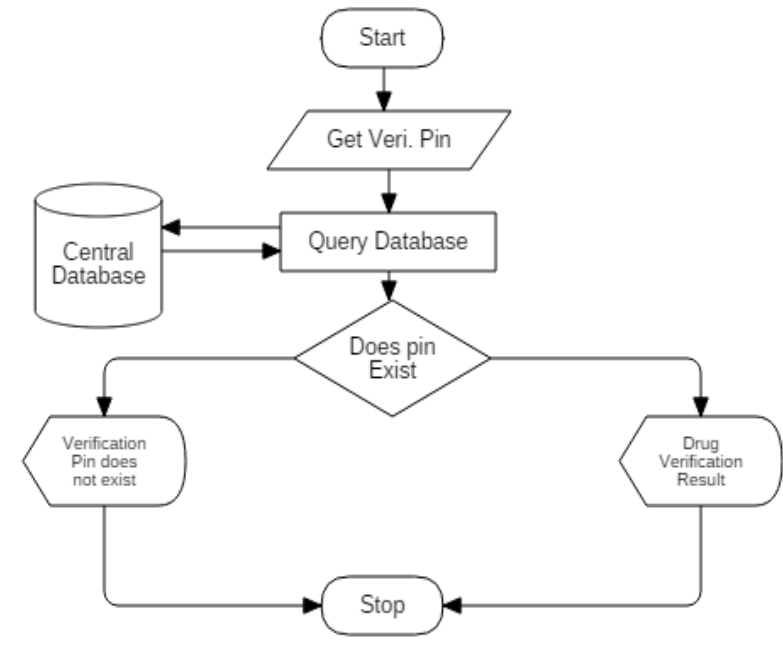

Fig. 2. Flowchart for NAFDAC MAS.

This system is a drug verification system built on the android operating system. This works in parallel with a database of drug manufacturers, drugs and drug verification pins representing the NAFDAC database hosted on a server online, from where the mobile application queries to supply user with the authenticity information of a given drug.

The NAFDAC database is populated with data from a NAFDAC admin web application. This web application is deployed to do the following:

Drug Manufacturers Registration

Drug Registration

Drug Verification Pin Generation

Drug Registration Slip Printing

Overview of Manufacturers

Overview of Registered Drugs

High level model of the developed system Fig. 3 shows a high level model of the developed system.

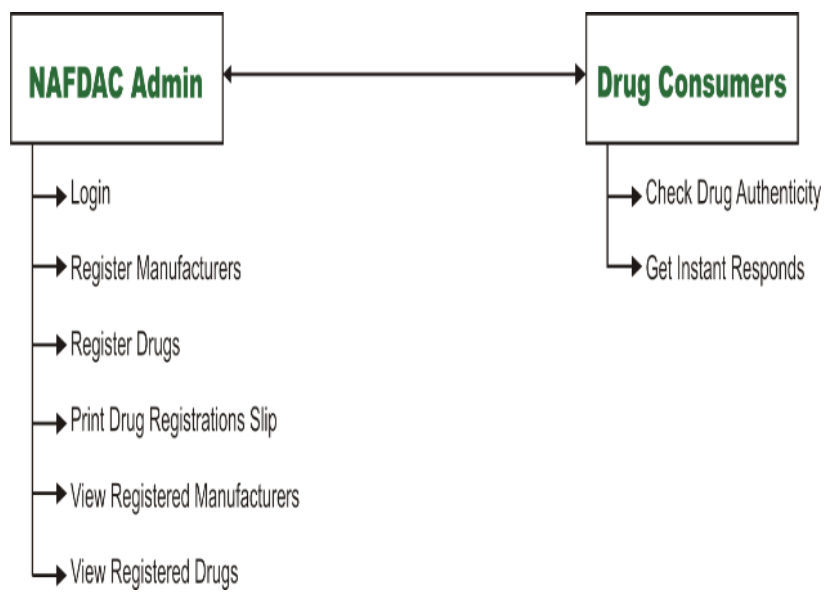

Fig. 3. High Level Model of the Developed System. 


\section{SYSTEM ARCHITECTURE}

A set of artifacts (that is: principles, guidelines, policies, models, standards, and processes) and the relationships between these artifacts, that guide the selection, creation, and implementation of solutions aligned with business goals [9]. Fig. 4 is a system architecture diagram of the developed NAFDAC VERIFY which is meant to partner with other platforms for effective drug verification.

\section{A. Use Case Diagram}

Use Case Diagram is used to represent how actions are performed by the different characters and to whom/what the actions are performed. It is a mimic of the use of the system in real-time. Fig. 5 shows the interaction between actors/users of the system and the system itself.

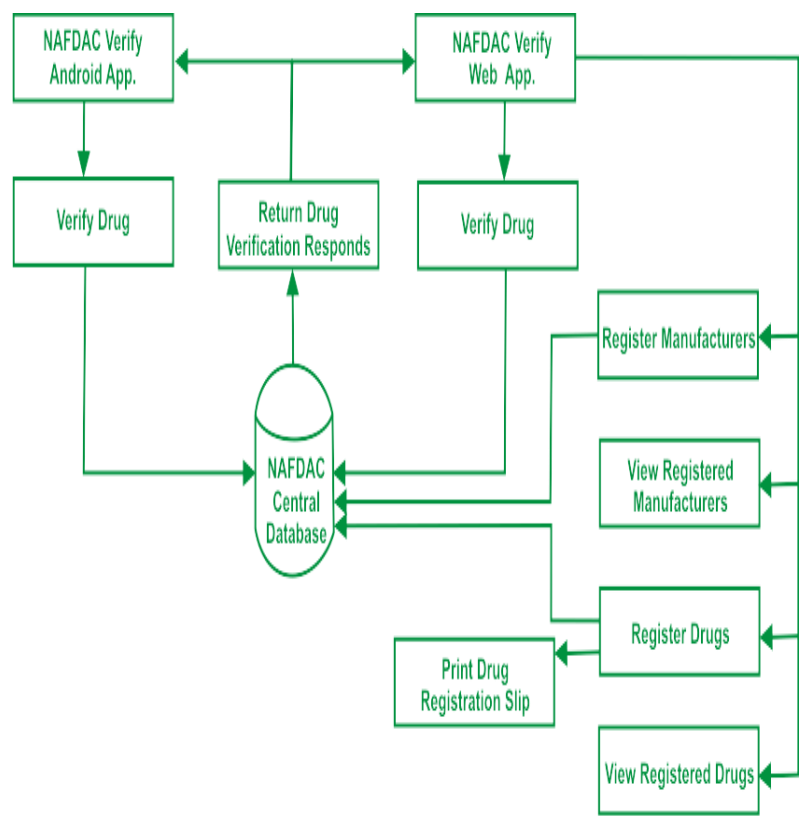

Fig. 4. System Architecture Diagram of the NAFDAC Drug Verification System.

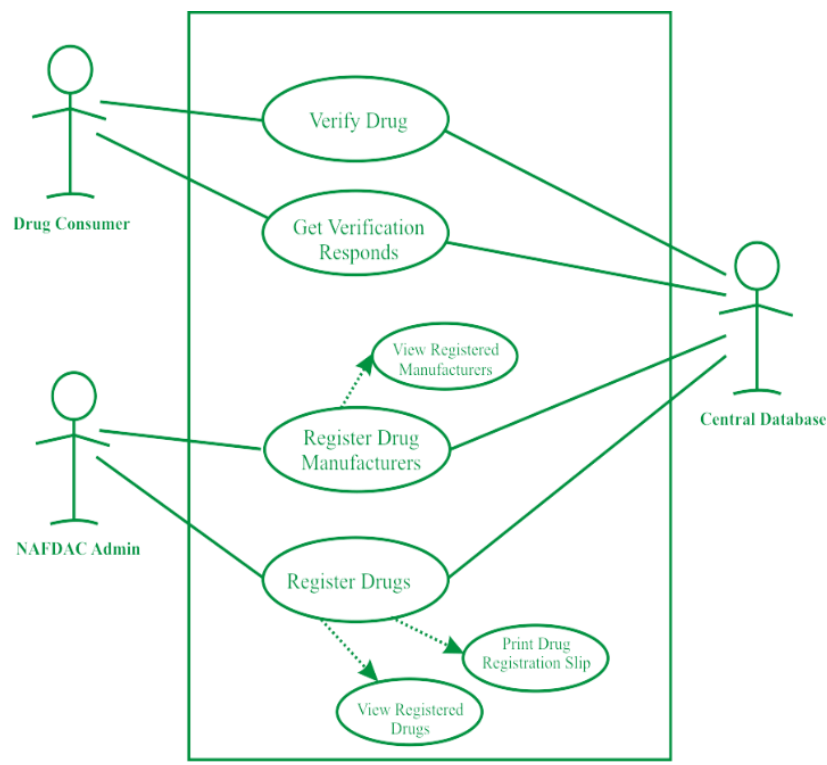

Fig. 5. The use Case Diagram of the System
1) Entity Relationship Diagram (ERD): Entity Relationship Diagram as shown in Fig. 6 provides a clear view of the relationships among the different entities associated with the system. This gives one idea of the flow of action performed by the system entities.

\section{B. Menu Design}

The Home Page of the system is depicted in Fig. 7 while Fig. 8 shows the process decomposition of the drug verification system and provides a navigation to several aspects of the system that work in synchronization with each other to achieve the main aim.

This menu serves as an authentication to NAFDAC admin panel. The authentication was implemented using PHP and MYSQLi. This menu is most concerned with security, therefore certain precautions were considered such as preventing Structured Query Language (SQL) injection. SQL injection is a malicious use of the SQL statement in a form. The attacker consciously login using a database query, this may grant them access to a system is the query successfully implement.

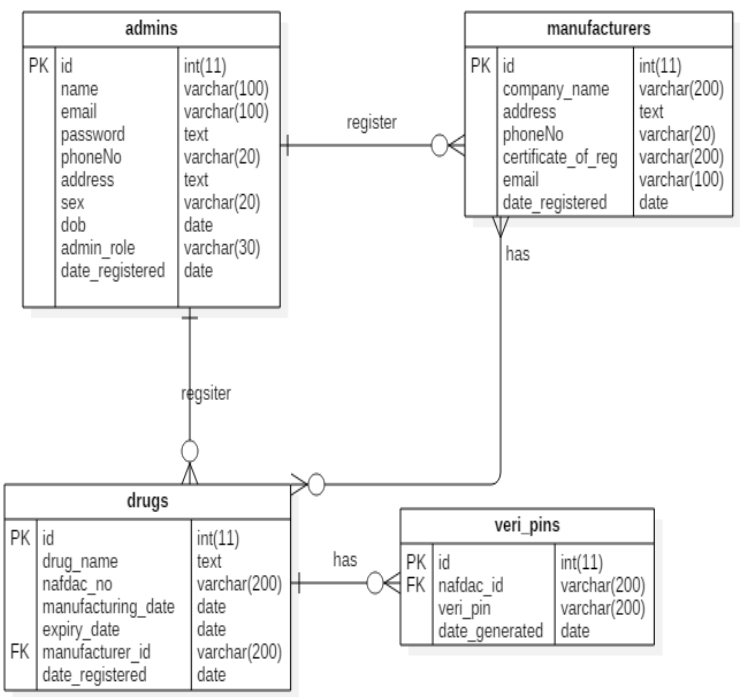

Fig. 6. Entity Relationship Diagram (ERD) of the New System.

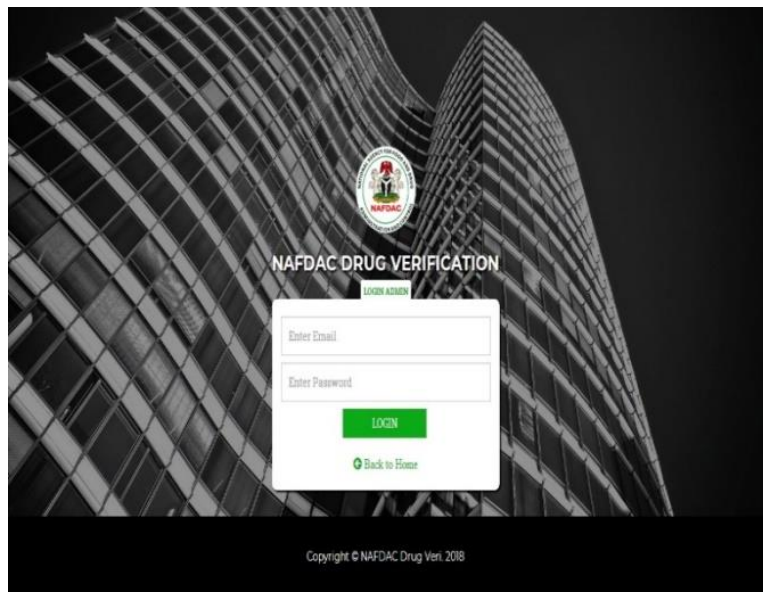

Fig. 7. The Admin Login Page for NAFDAC Staff in Order to Register New Drugs. 


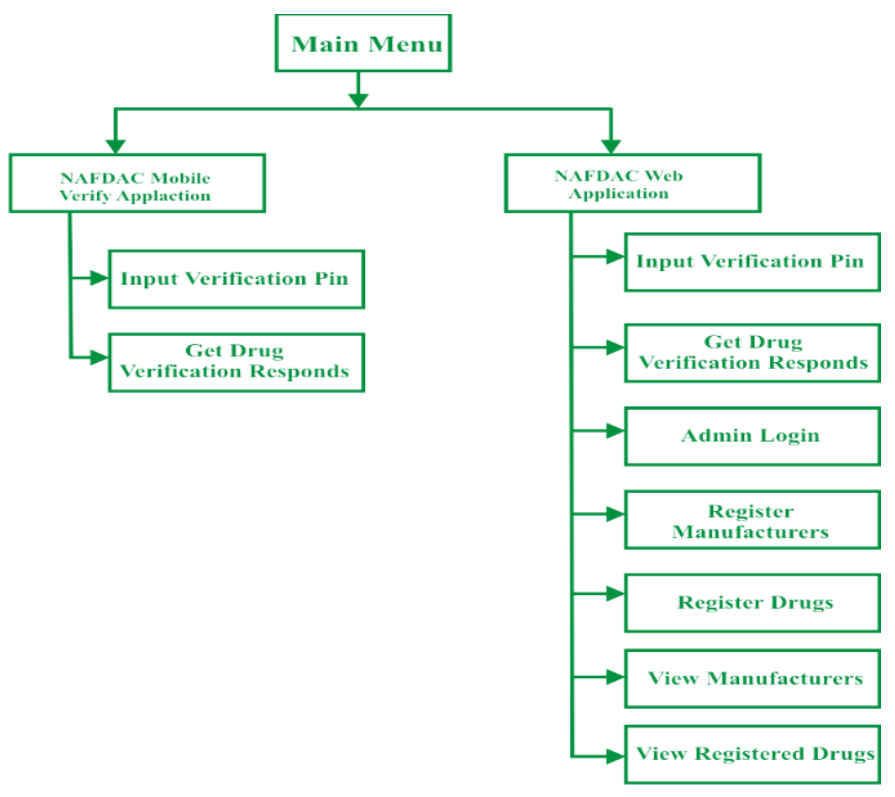

Fig. 8. Main Menu Design Showing the Functionalities of the Systems.

2) Subsystem design: The entire system is made up of subsystems whose functionalities help to achieve the goal of the system. The subsystems include:

a) The Android Platform Sub System as shown in Fig. 9 is built to get input data into the system in the form of verification PIN. It runs on the Android Operating System.

b) The Web Platform Subsystem as shown in Fig. 10 is designed for both drug verification and the NAFDAC Database Management System.

3) Program module design: The proposed system is made up of several modules which make up the entire system. Few of these include:

- Admin Login Module

The login module is based on the web application. It serves as a security restriction to the NAFDAC database operation. It is an access to the following admin privileges:

a) Drug manufacturers registration

b) Drug registration

c) Overview of registered drug manufacturers

d) Overview of registered drugs

e) Printing of drug registration slip containing drug verification pins.

f) Reprinting of drug verification pins for a given drug.

- Android Drug Verification Module

This module is based on the android operating system. It supports drug consumers who choose to use the android application for drug verification. It also queries the central database if the inputted drug verification pin is valid, if valid it gives the entire details about the drug including the manufacturer name, NAFDAC number, manufacturing date, expiry date, originality status (expired, original); else if it is not valid it gives a pin not found responds.

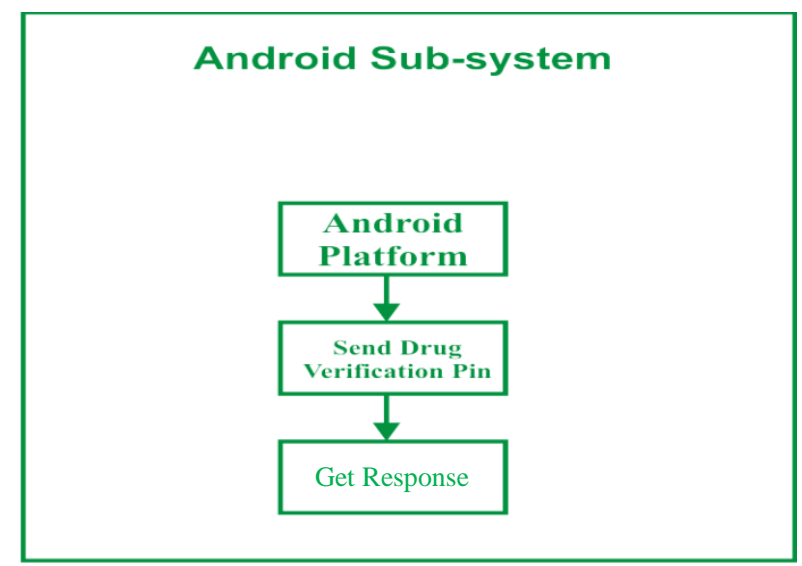

Fig. 9. The Android Platform.

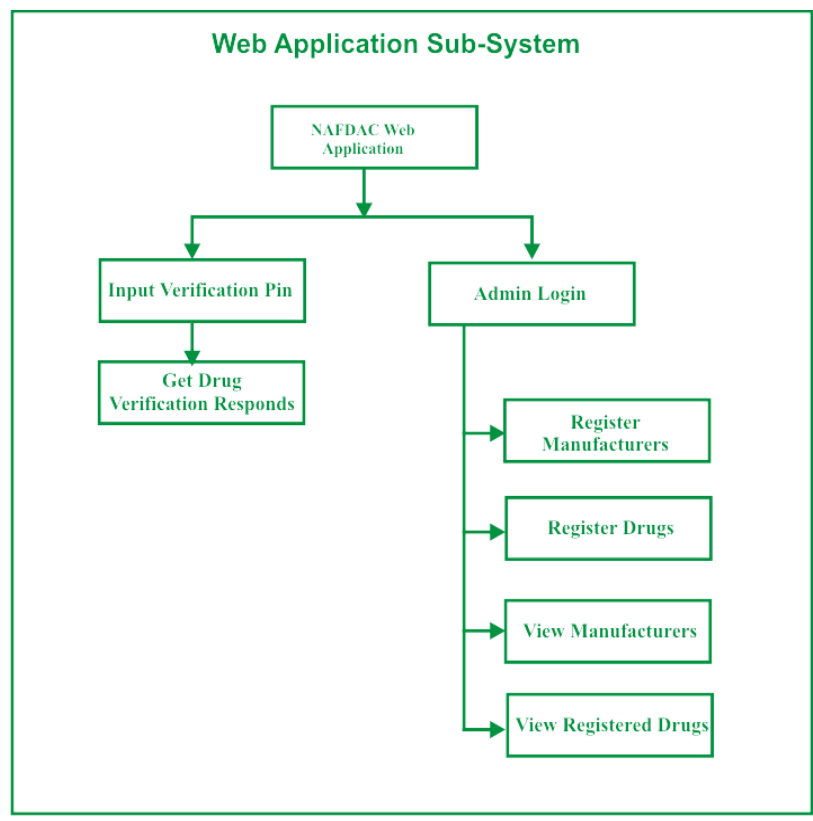

Fig. 10. The Web Platform.

4) Mobile drug verification menu implementation: The mobile drug verification menu shown in Fig. 11 was designed to enable drug verification PIN collection and initiate a query to the central database. This was implemented using java as a programming language on the android operating system. The HttpURLConnection was used to pass the verification pin data through the web hosted Application Programming Interface (API) http://mirarticles.com/nafdac /api/verify.php. The API processes the verification pin and queries the central database, then returns responds to the android application for display.

\section{Register Drugs Menu Implementation}

This is a menu that enables the NAFDAC admin do a drug registration. It was designed to accept inputs such as drug name, number of drugs, NAFDAC number, manufacturing date, expiry date and manufacturer. When a registration is initiated through the register button, a drug verification pin is generated following a predefined algorithm. 


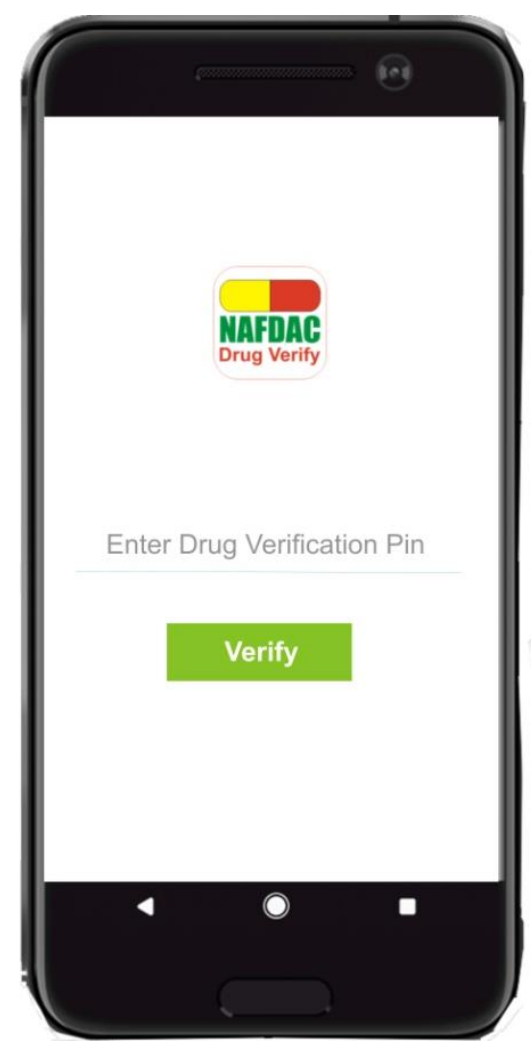

Fig. 11. Mobile Drug Verification Menu Implementation.

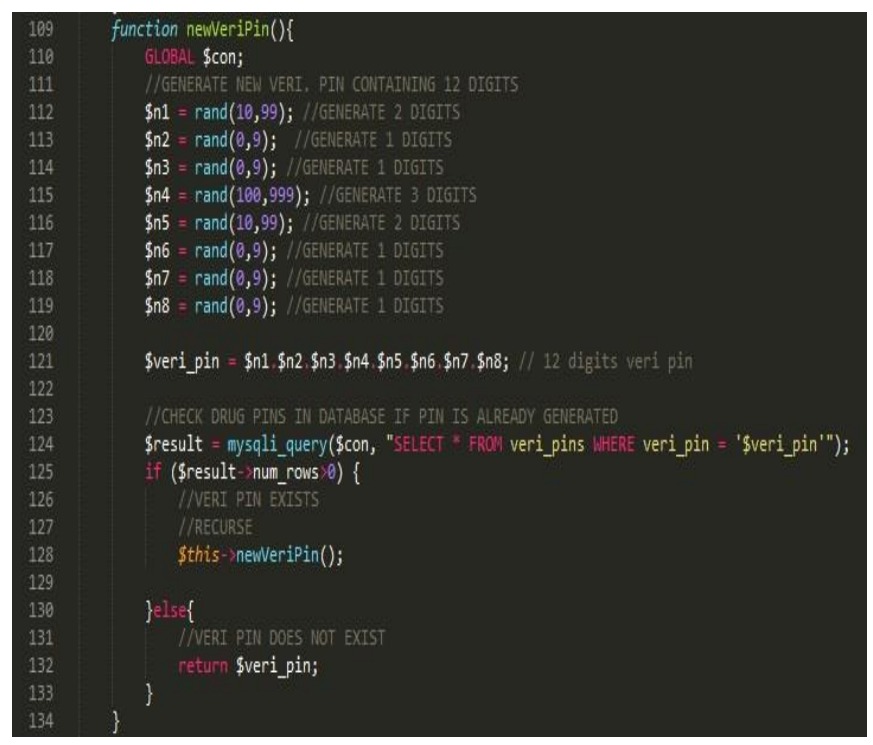

Fig. 12. The Drug Verification Pin Generation Program Code.

The code, part of which is shown in Fig. 12 comprises a concatenation of 8 variables, each generating random numbers. n1, n2, n3, n4, n5, n6, n7, n8 generates 2, 1, 1, 3, 2, $1,1,1$ digit(s) respectively, this forms a total of 12 randomly generated digits called the verification pin. From line 124 of the code, the generated PIN is checked if it already exists to avoid duplication of the same PIN. If it already exists, the function will call itself again in line 128. The flowchart for the drug verification PIN generation is shown in Fig. 13.

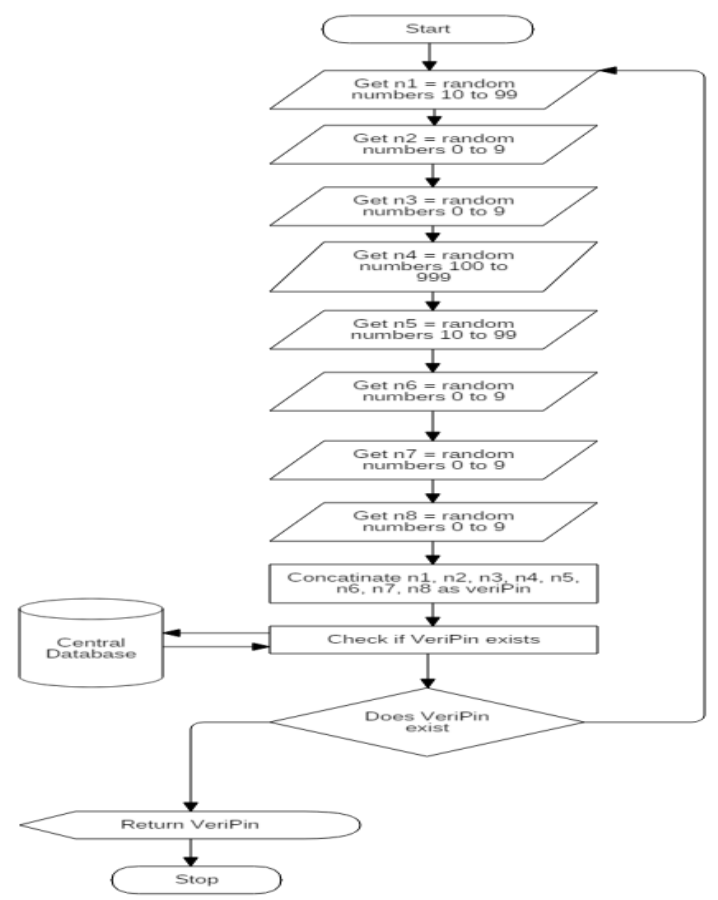

Fig. 13. Flowchart Diagram for Drug Verification PIN Generation.

\section{Subsystem Implementation}

1) The mobile drug verification subsystem: The mobile drug verification subsystem is a part of the entire drug verification application which was designed to enable drug verification pin collection and initiate a query to the central database. This was implemented using java as a programming language on the android operating system. The HttpURLConnection was used to pass the verification pin data through the web hosted Application Programming Interface (API) http://mirarticles.com/nafdac/api/verify.php. The API processes the verification pin and queries the central database, then returns response to the android application for display. Fig. 14 shows the drug verification API code.

2) The web subsystem implementation: The web subsystem was implemented and designed using HTML, CSS, JavaScript, PHP and MYSQL.

3) Program module implementation: In this section we paid more attention on the underground implementation of the program module: Android Drug Verification Module.

4) Android drug verification module implementation: The drug verification pin was collected using the android method called findViewById, and in precision we used veripin = (EditText) findViewById(R.id.veri). veri is the id given to the drug verification pin input field in the android application. The data was immediately authenticated if it is empty using the java code Veripin.trim().equals(""). If empty, an android toast is made to display an error message, else a class called Background which extends the AsyncTask predefined class was called. The AsyncTask helps some processes to run at the background of the application. These processes include using the java httpURLConnection to send the verification pin 
through the apihttp://mirarticles.com/nafdac/api/verify.php. Once a responds text is returned, it is sent to a new android activity class called Result.java. Here the result is interpreted and displayed to the user.

The response text is a string of results separated with "“". It looks like thisstatus|drug_name|nafdac_no|manufacturer_name |manufacturing_date|expiry_date|veri_pin. This result is interpreted and formatted for user's view.

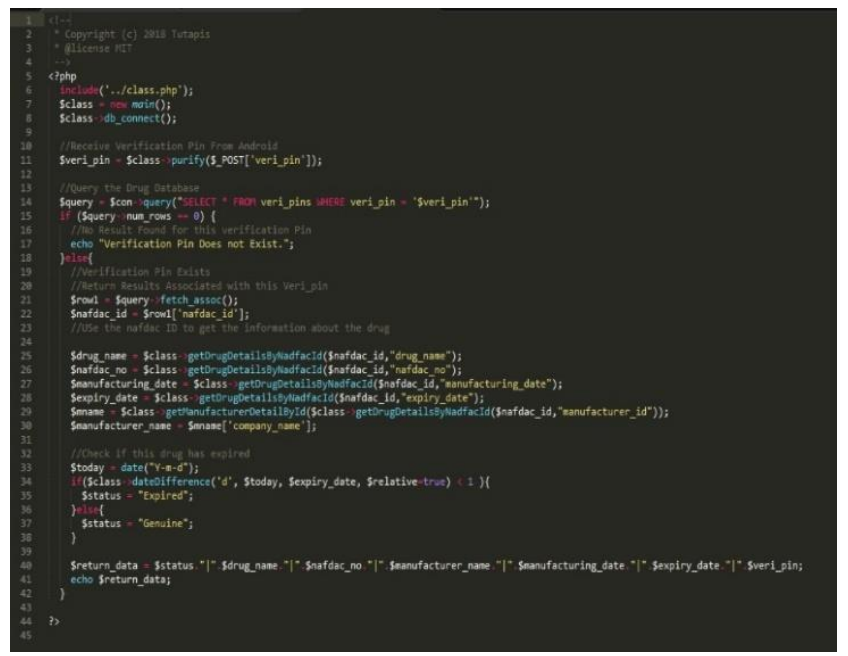

Fig. 14. The Drug Verification API Source Code.

\section{TEST RESULT}

These are outputs of the workability of the tested system. They give evidence of a fully implemented system when tested with real-time data. Fig. 15 and 16 shows a sample output for a genuine and expired drug verification on the web application respectively. Furthermore, Fig. 17 shows a sample output of registered drugs database with the registered PINs.

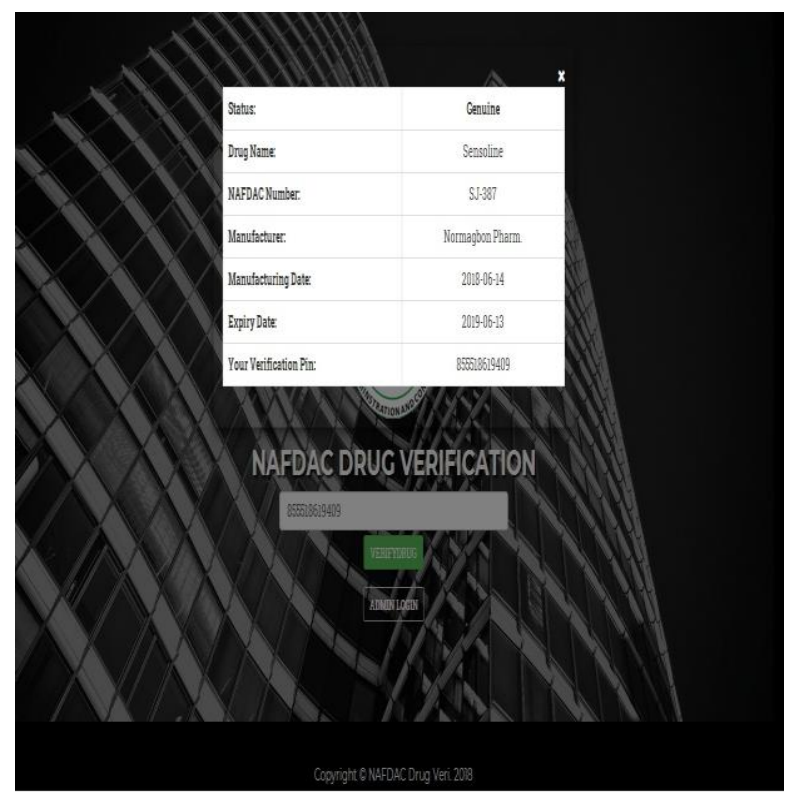

Fig. 15. An Output Sample for a Genuine Drug Verified on the Web Application.

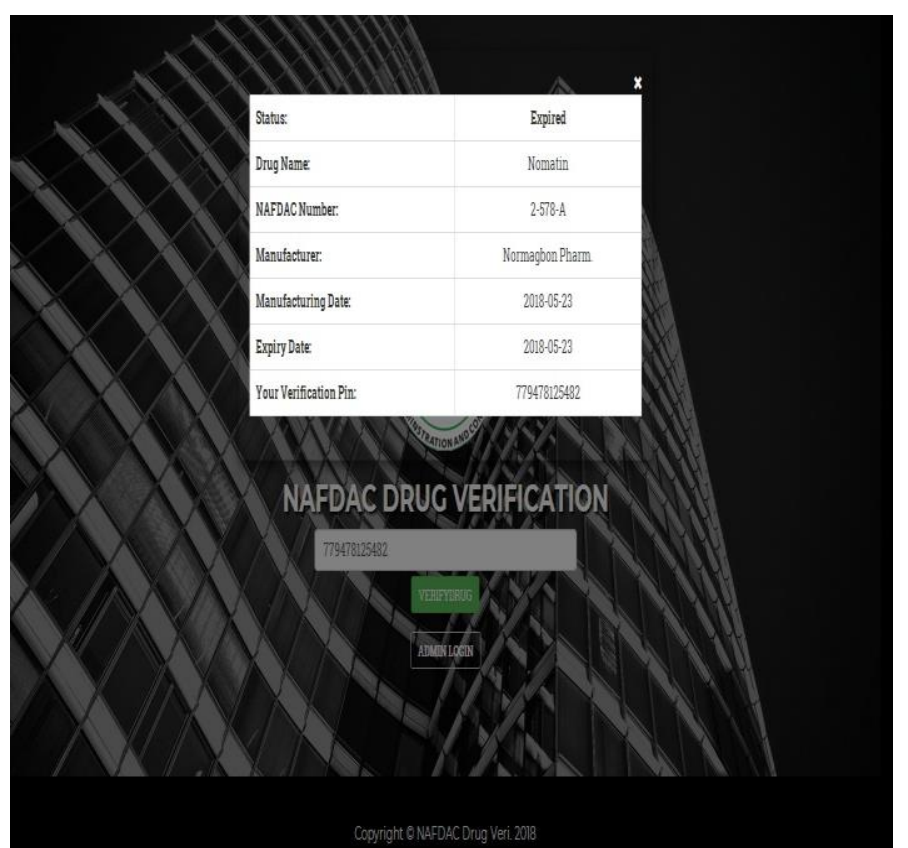

Fig. 16. An Output Sample for an Expired Drug Verified on the Web Application.

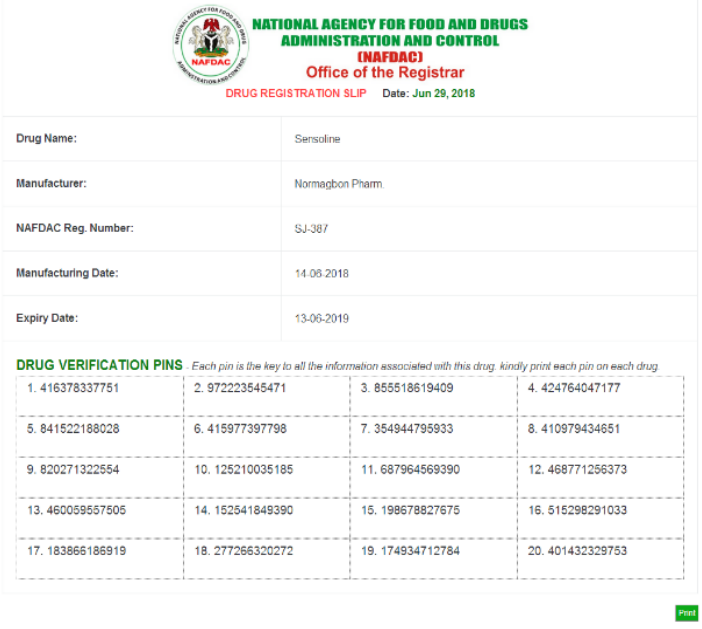

Fig. 17. Test Result for a Registered Drug with the Verification PINs.

\section{A. System Integration}

System integration as shown in Fig. 18 depicts how the various subsystems were bought together to form the main system. The following were integrated:

a) The Android Verification Application

b) The NAFDAC Web Application

c) The Central Database 


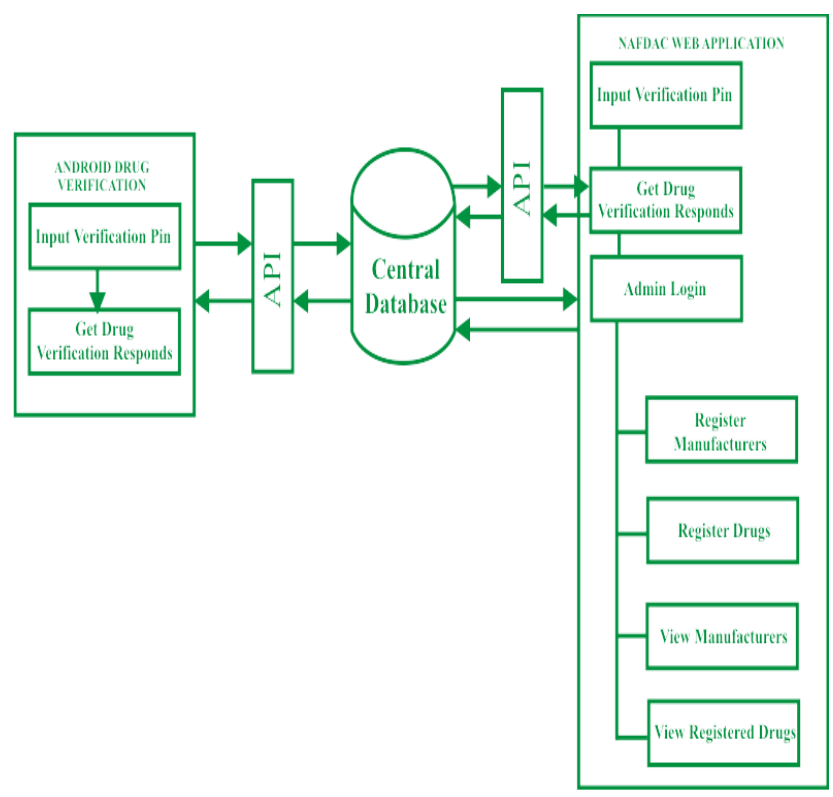

Fig. 18. An Integration of the System Subsystems.

\section{CONCLUSION}

The challenges of verifying fake drugs and the rampant distribution of expired rugs and beverages which cause hazard to the health of humans, led to this research. Despite the existence successive strategies to eliminate consumption of fake and expired drugs. The anchor points of the crime of fake and expired drug production distribution can be tackled by the introduction of a new drug verification system that is centered on absolute and effective verification of all drugs using an interactive mobile and web application. Therefore, the following were put in consideration during this research:

1) Verification beyond NAFDAC number, knowing that the correctness of a NAFDAC number does not declare the drug originality. It was discovered that many manufacturers print the NAFDAC number of an original drug and replicate same on fake ones, capitalizing on the reluctance of the consumers to scratch and verify.

2) Drug Information centered verification which pulls out the real information of the drug in the database for comparison with the one in question provides in-depth information such as if a drug has expired at the point of verification or if the verification pin does not exist.

3) To provide a real-time drug verification platform, this research has provided alternative means for consumers to verify the authenticity of their drugs including that of partnering with a third party technology company Sproxil@ that heralds the Mobile Authentication Service (MAS). This research was made to advance these existing strategies of the agency to ensure a consumer oriented drug verification in realtime and covers the loopholes of the preceding systems.

\section{REFERENCES}

[1] Olike, "The Fight Against Fake Drugs by NAFDAC in Nigeria," in 44th International Course in Health Development, Vrije Universiteit Amsterdam, 2007-2008.

[2] G. D. Berverly, "Counterfeit Drugs and Medical Devices in Developing Countries," Dove Press, vol. V, pp. 11-22, 2014.

[3] A. Olusegun, "Counterfeit drugs in Nigeria: A threat to public health," African journal of Pharmacy and Pharmacology, vol. VII, no. 36, pp. 2571-2576, 2013.

[4] O. Y. Buowari, "Fake and Counterfeit Drug: A review," AFRIMEDIC Journal, vol. 3, no. 2, pp. 1-4, 2012.

[5] A. C. Emmanuel, Deborah, O.O. and E. N. Gloria, "Food And Drug Counterfieting In The Developing Nations; The Implications and WayOut," Academic Research International, vol. III, no. 2, pp. 24-31, 2012.

[6] Impact, Impact: International Medical Products Anti-Counterfeit Taskforce, Roma: Agenzia, Roma: Intaliana del Farmaco, 2006.

[7] C. Obinna and G. Oluwale, "NAFDAC introduces new anticounterfeiting technologies Nigeria," Nigerian Vanguard, Lagos, 2010.

[8] J. A. Osuolale, "Institut Francais de Recherch Afrique/French Institue for Research in Africa," 2015. [Online]. Available: https://www.ifranigeria.org/files/44/Habitele/22/Joseph-Ayodokun:-Identification-drugsMAS.pdf. [Accessed 31 May 2019].

[9] F. Jeane-Claude, "New York University," 2011. [Online]. Available: www.nyu.edu>jcf>session2. [Accessed 20 May 2019]. 